\title{
Unicystic Ameloblastoma of mandible . Aggresive treatment A myth or a need. Case report and Extensive review of literature
}

\author{
Handa H, Bailoor DN, Naidu G, Shrivastava K, Raghuvanshi V
}

\begin{abstract}
Ameloblastomas are benign tumors whose importance lies in its potential to grow into enormous size with resulting bone deformity. Unicystic ameloblastoma (UA) refers to those cystic lesions that show clinical, radiographic, or gross features of a mandibular cyst, but on histological examination show a typical ameloblastomatous epithelium lining part of the cyst cavity, with or without luminal and/or mural tumour growth. The clinical and radiographic features of UCA, its differential diagnosis, histopathology and current concepts of management have been discussed.

We report a case of which show varied clinical and radiographic features and which histopatholoIgicaly was unicystic variant and was aggressively treated rather than a conservative approach which is a trade mark of cystic ameloblastoma

Key words: Ameloblastoma, Unicystic ameloblastoma, Multilocular, Conservative treatment
\end{abstract}

\section{Introduction}

Swellings of mandible mainly occur due to odontogenic or non-odontogenic etiology. The most common tumor of odontogenic origin is ameloblastoma which develops from epithelial cellular elements and dental tissues in various phases of development. It is a slow-growing, persistent and locally aggressive neoplasm of epithelial origin, affecting the posterior area of lower jaw in $80 \%$ of cases. The concept of this tumour was first introduced by Robinson and Martinez in 1977. The other name as recognised by WHO is 'cystogenic ameloblastoma'. The relative frequency of occurrence of unicystic ameloblastoma has been reported as between $5 \%$ to $22 \%$ of all types of ameloblastomas. ${ }^{2}$ Generally attention has been directed on the surgical management of conventional, solid ameloblastoma with no delineated management protocols for the cystic variant. The management protocols, therefore range from the radical resection reserved for solid ameloblastomas to simple enucleation generally considered apt for a dentigerous cyst. ${ }^{3}$

\section{Case Report}

A 23-year-old female reported in the outpatient department with the chief complaint of asymptomatic firm swelling on left jaw since 1 month which gradually increased to current size.

Dental and medical history was insignificant while general examination also appeared to be normal.

On clinical examination, there was evidence of a solitary swelling about $3 \times 4 \mathrm{~cm}$ on left side of mandible seen extending superoinferiorly- $0.5 \mathrm{~cm}$ below ear lobe and $1 \mathrm{~cm}$ ahead of angle of mandible and anteroposteriorly- from left commisural lip to angle of mandible. Overlying skin was normal in color. Swelling was non tender, firm and non compressible.(fig1)

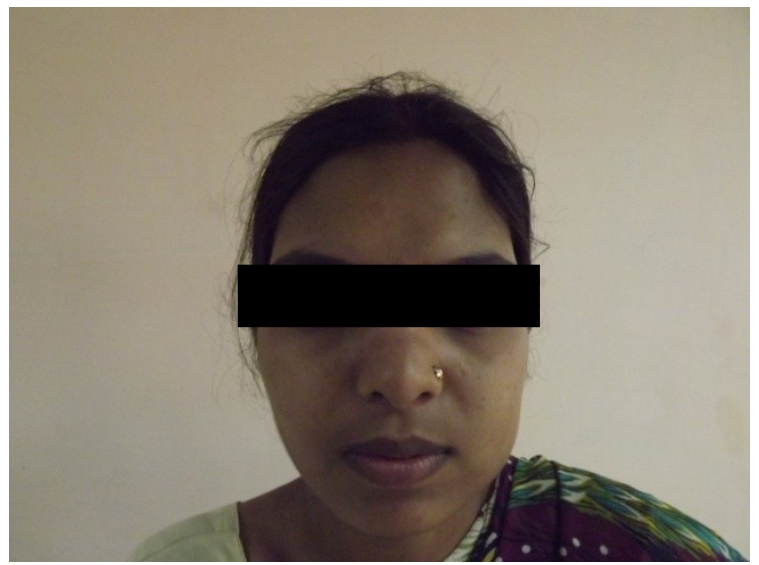

FIGURE 1 showing Extraoral swelling on left side of face 
Submandibular lymph nodes were palpable and non tender on the left side . Intraoral examination revealed a painless swelling in the left posterior mandibular vestibule from right mandibular first premolar to the distal aspect of second molar on the same side. The swelling was firm, non tender with fluctuance noted with superior aspect of swelling in relation to 47 and was covered by normal mucous membrane. Buccal cortical expansion was found. (fig 2)

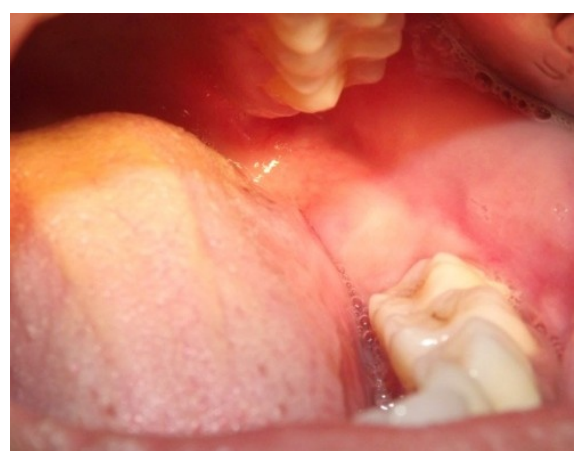

FIGURE 2 showing intraoral vestibular obliteration

Aspiration cytology was performed and The lesion was perforated with a wide bore needle from the region of 47 where fluctuance was present. On aspiration"Sanguinous aspirate" about $3 \mathrm{ml}$ was obtained(fig3).

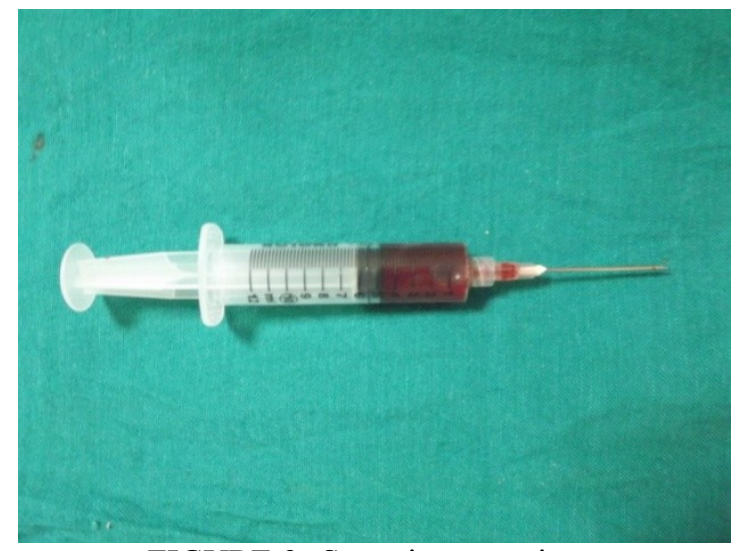

FIGURE 3 -Sanguinous aspirate

Panoramic radiograph reveals multilocular radiolucent lesion of about $4 \times 3 \mathrm{~cm}$. extending antero- posteriorly from mesial aspect of 37 to the angle of mandible Superoinferiorly from the ramus area to lower border of mandible. Internally incomplete indistinct septa present suggestive of pseudoloculations .Superiorly and inferiorly margins were indistinct with sclerotic border noted posteriorly. Thinning of lower border of mandible is evident. Also resorption of distal root of 37 was noted.(fig4)

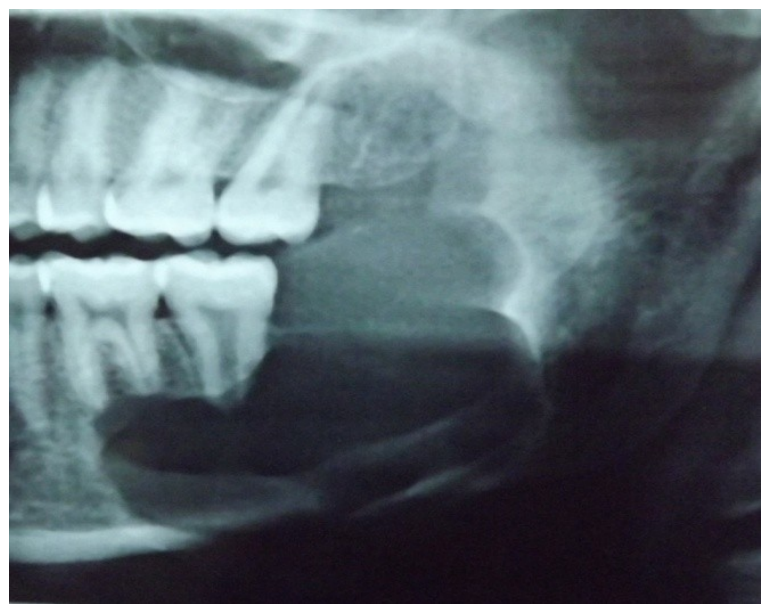

FIGURE 4-Radiolucent lesion with pseudoloculations noted on left side of mandible 
Considering the site, age and it being multilocular along with blood aspirate. Differential diagnosis of ameloblastoma, odontogenic keratocyst,aneurysmal bone cyst and central hemangioma and central giant cell granuloma was considered.

CT scan was done and revealed the same lesion with An expansile osteolytic lesion involving the left side of mandible and ramus region with internally areas of hypodensity noted. Superiorly some areas of contrast enhancements is present which suggestive of vascularity. (fig5).

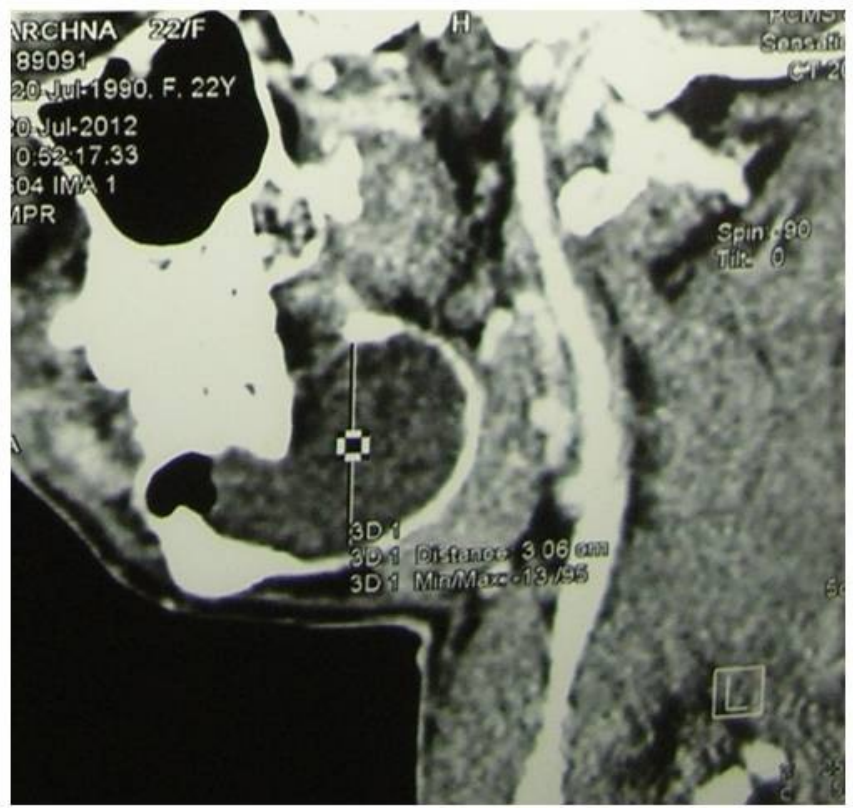

FIGURE 5 Sagittal section expansile lesion areas of hypodensity present internally within the lesion.

Axial CT reveals a soft tissue window. An osteolytic expansile lesion having dimensions of 4.47 anteroposteriorly and mesiolaterally $2.49 \mathrm{~cm}$ (FIG 6).Coronal CT reveals an osteolytic expansile lesion having a diagonal dimension of $4.74 \mathrm{~cm}$. Superiorly some areas of contrast enhancements is present which suggestive of vascularity( FIG 7).CT Angigography was performed to check for the major feeder vessels involved but CT Angio revealed no vascular involvement of pathology was noted. (FIG 8 ).

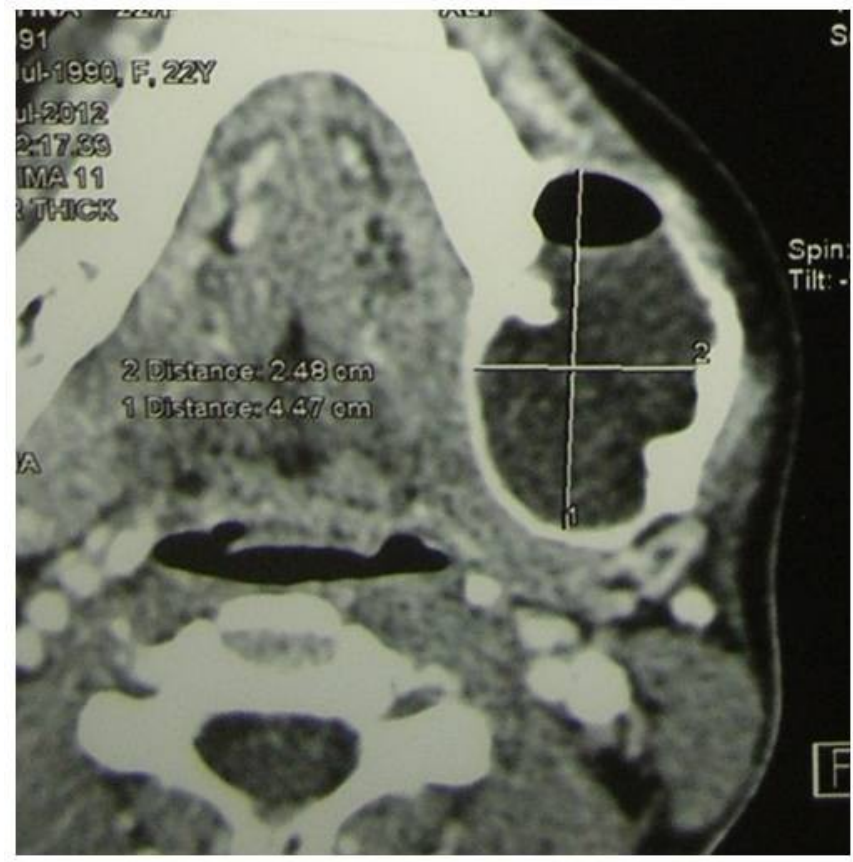

FIGURE 6 Axial CT 


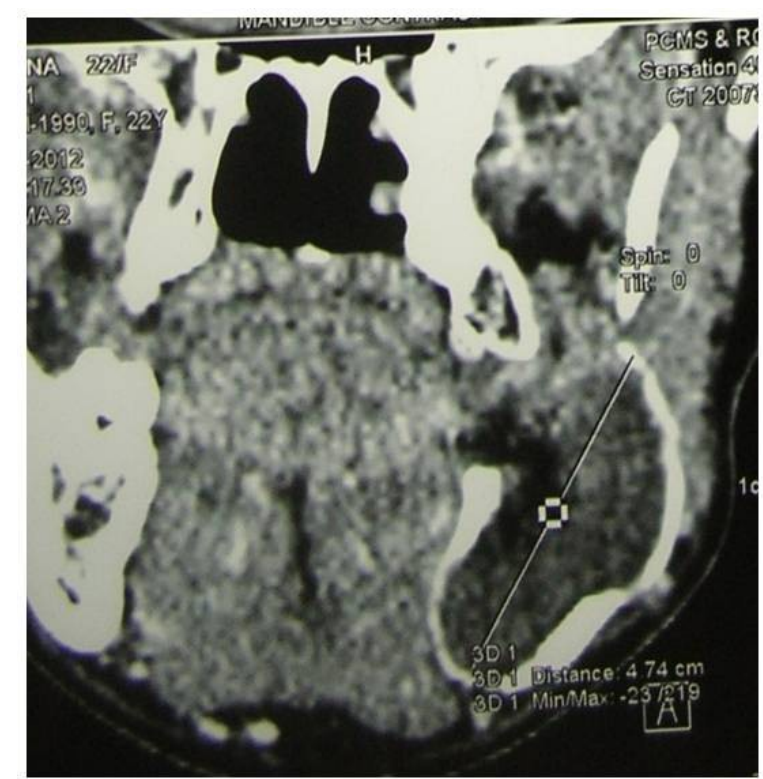

FIGURE 7 CORONAL CT

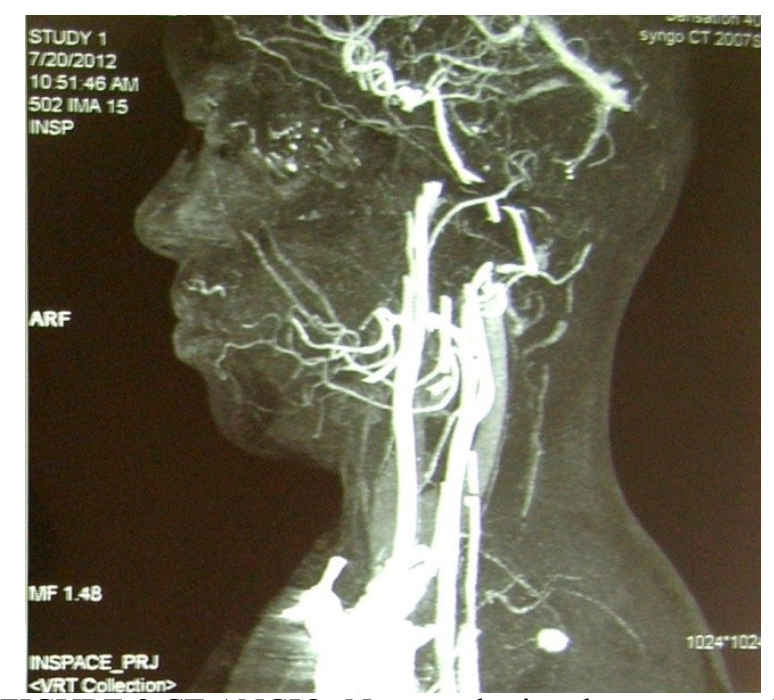

FIGURE 8 CT ANGIO- No vascular involvement noted

Segmental resection (mandibulectomy) was done under general anaesthesia followed by primary reconstruction with anterior ileac non vascularized bone graft and the specimen was subjected to histopathological examination.(fig9)

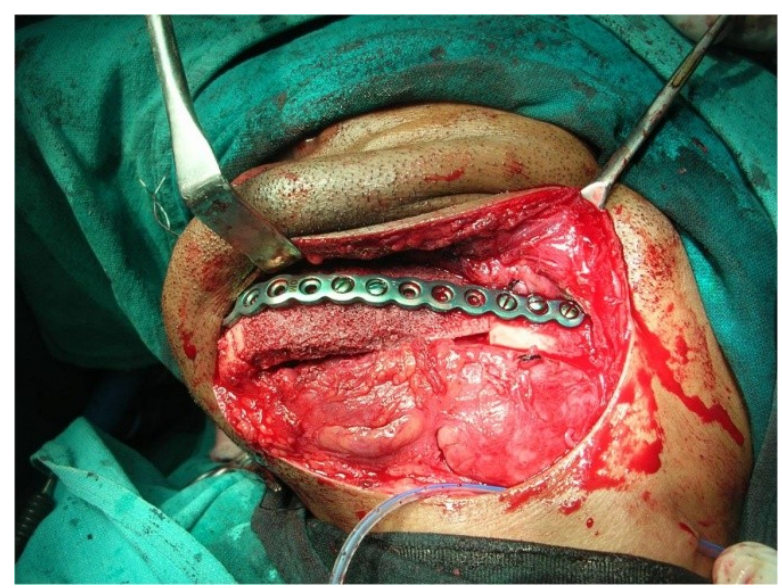

FIGURE 9-Surgical photo showing en block resection 
Histopathological findings reveal cystic lumen with ameloblasti cepithelium. The epithelial lining of the lumen is uniform in thickness and has a slightly hyperchromatic layer of palisaded basal cells. The remaining layers resemble stellate reticulum. The connective tissue consist of mild inflammatory cell response. Based on these findings a diagnosis of Unicystic Ameloblastoma (luminal)was made.(fig10)

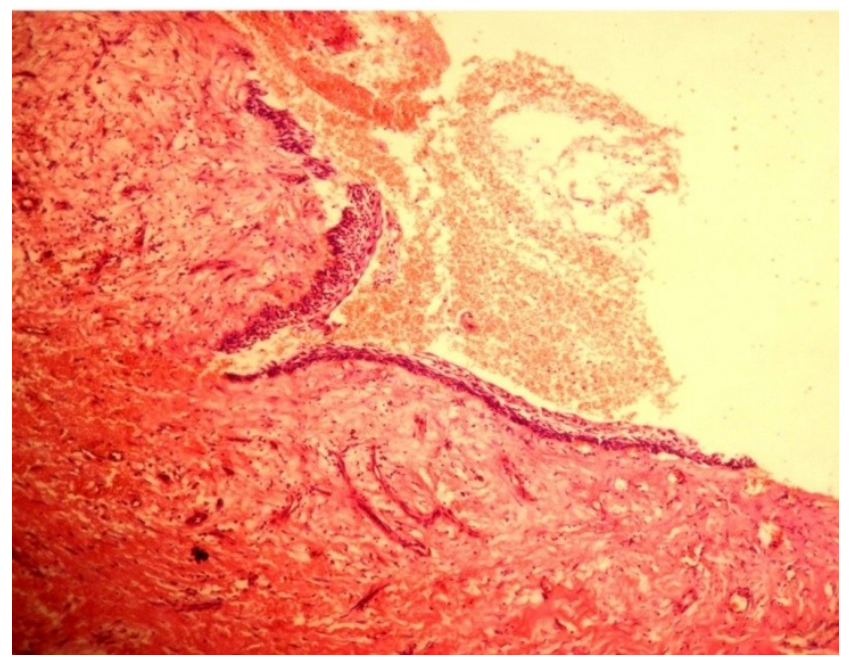

FIGURE 10- (left lower H\&E X 400).Proliferated ameloblastic epithelium

\section{Discussion}

Unicystic ameloblastoma is a rare type of ameloblastoma, accounting for about $6 \%$ of ameloblastomas . About $50 \%$ of the cases occur in the second decade of life. The mandible is affected more often than the maxilla. ${ }^{4}$

Patients most commonly present with swelling and facial asymmetry, pain being an occasional presenting symptom

Small lesions are sometimes discovered more on routine radiographic screening examinations or as a result of local effects (like tooth mobility, occlusal alterations and failure of eruption of teeth) produced by the tumor. $^{5}$

Between 50 and $80 \%$ of cases are associated with tooth impaction, the mandibular third molar being most often involved. ${ }^{5}$ In the present case swelling $\mathrm{n}$ was present at the third molar region,asymptomatic firm swelling with fluctuance noted with superior surface with 47 and "sanguinous" aspirate was obtained. In the most comprehensive study on the radiographic aspects of UCA, it was found that the unilocular: multilocular ratio was 13:3 for cases associated with impacted teeth, against an almost equal 8:7 for the 'non-dentigerous' types. $^{6}$

In our case report incomplete septa were noted giving an appearance of multiloculations with well defined sclerotic borders noted posteriorly, CT scan too revealed superiorly contrast enhancement which is suggestive of vascularity and anteriorly cystic cavity along with post enhancement noted. CT angio in our case confirmed the non vascular involvement.

If during enucleation a mural mass is discovered, the surgeon should flag it with sutures to enable the pathologist to concentrate on it as an area of greatest concern. An intraoperative decision can then be made to treat the bony cavity with either Carnoy's solution or liquid nitrogen cryotherapy. ${ }^{7}$

Histologically, the minimum criteria for diagnosing a lesion as UA is the demonstration of a single cystic sac lined by odontogenic (ameloblastomatous) epithelium often seen only in focal areas. Ackermann et al in their landmark study classified the histological subtypes into three patterns, namely luminal (type 1), intraluminal (type 2) and mural patterns (type 3).

UA is believed to be less aggressive and responds more favourably to conservative surgery than the solid or multicystic ameloblastomas. ${ }^{9}$ Cryotherapy with liquid nitrogen has also been suggested after enucleation and curettage to be an effective method of treatment of these lesions. ${ }^{10}$ But in the present case because of varied radiographic features which was presented as cystic tumor and vascular lesion as well as thinning of mandible we planned resection of mandible rather than conservative treatment. Although in cases of unicystic ameloblastoma conservative approach is the treatment of choice but,we considered the age of patient,extent of lesion and widespread involvement of lower border of mandible and planned an aggressive treatment(resection) of mandible.Recurrence is also related to the histologic subtypes, among which those invading the fibrous wall have a rate of $35.7 \%$, but others have a rate of only $6.7 \%{ }^{11}$ UAs are considered to be 
less aggressive form of ameloblastomas and can be successfully removed by simple enucleation or less aggressive surgery.

The use of Carnoy's solution to decrease chances of recurrence after conservative surgical treatment of UCAs was initially suggested by Stoelinga and Bronkhorst in $1988^{12}$

\section{Conclusion}

Both clinical and radiologic findings share equal contribution to the final diagnosis. Radiographs are an important aid for the diagnosis of oral lesions of various types, especially those that involve bone. This case also highlights the importance of careful examination,radiographic evaluation and detailed observation of the entire specimen and the usefulness of deeper sections in diagnosis of unicystic ameloblastoma and an ideal and meticulous treatment so as to prevent recurrence. Thus, it is of utmost importance to correlate histopathologic findings with clinical and radiographic features to achieve at a correct definitive diagnosis as all such lesions may have prognostically different biologic behaviours and the final diagnosis may alter the therapeutic decision significantly.

\section{References}

[1]. WHO Classification of Tumours Series. In: Barnes L, Everson JW, Reichart P, Sindransky D. Pathology and genetics of head and neck tumous. Lyon: IARC Press, 2005;296-300.

Reichart PA, Philipsen HP: Unicystic ameloblastoma. Odontogenic tumors and allied lesions. 1st Edn.;

[2]. Quintessence Publication. Co. Ltd London, 2004; pp77-86.

[3]. Mohammad yunus, unicystic ameloblastoma: a distinct clinicopathologic pakistan oral \& dental journal june 2009.vol 29; no. 1

[4]. Ramesh RS, Manjunath S, Ustad TH, Pais S, Shivakumar K. Unicystic ameloblastoma of the mandible - an unusual case report and review of literature. Head Neck Oncol 2010; 14;2:

[5]. Roos RE, Raubenheimer EJ, van Heerden WF: Clinico-pathological study of 30 unicystic ameloblastomas. J Dent Assoc S Afr 1994, 49:559-62.

[6]. Eversole LR, Leider AS, Strub D. Radiographic characteristics of cystogenic ameloblastoma. Oral Surg Oral Med Oral Pathol 1984; 57: $572-77$

[7]. Wood NK, Kuc IM. Pericoronal radiolucencies. In: Wood NK,Goaz PW, eds. Differential diagnosis of oral and maxillofacial lesions. 5th edition. St. Louis: Mosby. 1997

[8]. Ackerman GL, Altini M, Shear M. The unicystic ameloblastoma: a clinicopathologic study of 57 cases. J Oral Pathol 1988; 17: 54146

[9]. Robinson L, Martinez MG. Unicystic ameloblastoma: A prognostically distinct entity. Cancer 1977;40:2278-85.

[10]. Pogrel MA. The management of lesions of the jaws with liquid nitrogen cryotherapy. J Calif Dent Assoc 1995; 23: 54-57

[11]. $\mathrm{Li} \mathrm{T}, \mathrm{Wu} \mathrm{Y,} \mathrm{Yu} \mathrm{S,} \mathrm{Yu} \mathrm{G.} \mathrm{Clinicopathological} \mathrm{features} \mathrm{of} \mathrm{unicystic} \mathrm{ameloblastoma} \mathrm{with} \mathrm{special} \mathrm{reference} \mathrm{to} \mathrm{its} \mathrm{recurrence.}$ Zhonghua Kou Qiang Yi Xue Za Zhi. 2002;37:210-2

[12]. Stoelinga PJW, Bronkhorst FB. The incidence, multiple presentation and recurrence of aggressive cysts of the jaws. J Craniomaxillofac Surg 1988; 16: 184-95 